\title{
Results from the first burst hardware injections performed on GEO 600
}

\author{
R Balasubramanian ${ }^{1}$, H Grote ${ }^{2}$, I S Heng ${ }^{2}$, M Hewitson ${ }^{2}$, H Lück²,

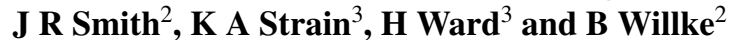 \\ ${ }^{1}$ Department of Physics and Astronomy, Cardiff University, PO Box 913, Cardiff, CF2 3YB, UK \\ 2 Max-Planck-Institut für Gravitationsphysik (Albert-Einstein-Institut) und Universität Hannover, \\ Callinstr 38, D-30167 Hannover, Germany \\ ${ }^{3}$ Physics and Astronomy, University of Glasgow, Glasgow G12 8QQ, UK \\ E-mail: R.Balasubramanian@astro.cf.ac.uk,martin.hewitson@aei.mpg.de and \\ siong@aei.mpg.de
}

Received 22 March 2005, in final form 27 May 2005

Published 4 July 2005

Online at stacks.iop.org/CQG/22/3015

\begin{abstract}
GEO 600 is a $600 \mathrm{~m}$ arm-length, laser interferometric gravitational wave detector, located about $25 \mathrm{~km}$ from Hannover, Germany. Starting in November 2003, GEO 600 took part in a coincident data taking period with other detectors around the world. During this time, GEO 600 acquired three weeks of 'science quality' data. These data are currently being processed to search for gravitational wave signatures. One such search is that for burst gravitational waves. To investigate the performance of any burst-search codes used to analyse the data, burst-like signals were injected into the detector after the data taking run; this was done by differentially driving the end mirrors of the interferometer. In this paper, we report on how the first burst hardware injections were performed on GEO 600, the type of signals injected and the analysis of the data from the period of injections. Results from the analysis are also presented and discussed.
\end{abstract}

PACS numbers: $95.55 . \mathrm{Br}, 95.75 . \mathrm{Kk}, 04.80 . \mathrm{Nn}, 95.55 . \mathrm{Ym}$

\section{Introduction}

The search for gravitational waves has been going on since the late 1960s when Weber built the first gravitational wave detector: a resonant-mass detector operating at room temperature. Today, there are several cryogenic resonant-mass gravitational wave detectors in long-term operation around the world which are several orders of magnitude more sensitive than earlier ones. These are ALLEGRO [1], AURIGA [2], EXPLORER and NAUTILUS [3]. In addition to these, a worldwide network of laser interferometric gravitational wave detectors is rapidly 
coming into operation. One of these is the British-German GEO 600 project [4] located near Hannover, Germany. In conjunction with the LIGO [5] and TAMA [6] detectors, GEO 600 acquired three weeks of scientifically useful data between 5 November 2003 and 13 January 2004; this data taking period is termed S3. Another interferometric gravitational wave detector, VIRGO [7] is currently still in the commissioning stage.

The data acquired during the science run are currently being analysed for possible signatures of signals from many different kinds of sources. One such analysis is the search for burst gravitational waves. Burst gravitational waves are defined as short, unmodelled pulses of gravitational radiation which could possibly be emitted from a catastrophic event such as a stellar core collapse.

A key step in the search for unmodelled bursts is to validate and characterize the analysis software. So far, various different search algorithms have been developed to try and detect burst-like signals in the data of gravitational wave detectors. This paper focuses on two of these algorithms: Excess Power and HACR (Hierarchical Algorithm for Clusters and Ridges). Other examples of burst-search algorithms are discussed in [8-10]. One desired validation step for these algorithms is to do hardware injections, that is, to inject a burst-like signal into the interferometer by introducing known differential arm-length changes. Such injections appear at the output of the interferometer in the main gravitational wave channel. Analysis of this data stream should lead to the recovery of the injected signals.

The first burst hardware injections in GEO 600 were performed on 16 and 17 January 2004. The recorded data have been analysed with two burst-search algorithms, Excess Power and HACR. In this paper, we report on the analysis of this first ever series of burst hardware injections at GEO 600. We begin with a description of how the injections were performed and a brief description of each analysis pipeline used. Slightly more emphasis is given to the details of the HACR algorithm, as this information is previously unpublished. The results of the two analyses follow, together with some concluding remarks.

\section{The injected events}

A period of a few hours was chosen shortly after the end of the S3 science run during which to perform hardware injections of burst-type signals. During this time, some parameters of the waveforms were varied, producing different sets of injected events. This section gives a description of the injected signals, together with a brief discussion of how the injections were performed.

\subsection{Generating the signals}

Injection of the burst-type signals can be viewed naturally as a four-step process. The first step is to use a particular waveform model to generate a gravitational wave strain signal. Having done this, the signal must be converted into a voltage that, when applied differentially to the Michelson length-control actuators, gives the differential displacement expected from such a waveform. The signal is then sent to the signal injection hardware and finally is applied to the length-control actuators of the Michelson control servo [11] to create the desired differential arm-length changes.

2.1.1. Signal waveform. The injected signals, in units proportional to strain, were sineGaussian waveforms of the form

$$
s(t)=A \cos \left(2 \pi f_{0} t\right) \exp \left(-\frac{(t-0.5)^{2}}{2 \tau^{2}}\right),
$$


where $A$ is a scaling amplitude, $f_{0}$ is the central frequency of the waveform and $\tau$ is related to the quality factor, $Q$, of the signal by

$$
\tau=\frac{Q}{2 \pi f_{0}} .
$$

The $0.5 \mathrm{~s}$ offset is included just to make the waveform peak in the middle of a $1 \mathrm{~s}$ data segment.

A digital representation, $s[n]$, of this waveform is produced by setting $t=n / f_{\mathrm{s}}$ where $f_{\mathrm{s}}$ is the required sample rate of the output signal.

Having created the digital signal (in units proportional to strain), we need to convert it into a force that we can apply differentially to the main test masses. The main lengthcontrol actuators used to keep the Michelson interferometer at its operating point can be used to induce a desired differential arm-length change in the interferometer. These actuators are located at the bottom of pendulum chains that suspend the main optics of the interferometer (see $[12,13]$ for details of the pendulums). For frequencies $f$ above the longitudinal resonance of these pendulums, the displacement of the test masses follows the signals applied to the actuator with an $f^{-2}$ response. In order to ensure that the resulting mirror displacement is directly proportional to the strain signal we calculated, we must filter the calculated signal with an $f^{2}$ response ('strain-to-force') before applying it to the actuators. This was done using a time domain, infinite impulse response (IIR) filter.

The signal generation routine, together with the strain-to-force filter, is implemented as a LabVIEW virtual instrument that communicates directly with the hardware injection system via a digital I/O card attached to the PC running the software.

As well as generating the signal for injection, the software is also responsible for controlling the parameter values that go into the calculation of each injected waveform. For the experiments described here, the following parameters were varied:

- overall strain amplitude, $A$,

- central frequency, $f_{0}$.

The injection of individual events into the detector was designed to occur at a random offset from the GPS second boundary. The injection times, together with the parameters used to produce each injected waveform, were stored to a file. As an additional record, the injected waveforms were recorded using the data acquisition system of GEO $[14,15]$. Due to the poor time accuracy of the PC clock, the injection times recorded to the file were not deemed accurate enough to do a comparative analysis. Therefore, the recording of the injected waveforms was subsequently used to determine the time of the peak of the waveform to an accuracy of around $60 \mu$ s (see sections 4.1.1 and 4.2.1).

2.1.2. Injection signal generator. In order to do temporally accurate waveform injections into the detector, a purpose-made injection hardware module was built [16]. The digital waveform to be injected is first downloaded, along with any relevant timing information, to the on-board RAM; the orchestration of this process is done by a Programmable Logic Chip in the module. The processor monitors timing signals provided by an external GPS unit and begins clocking out the waveform to a digital-to-analogue converter (DAC) at the precise offset from the 1 PPS (pulse-per-second) edge requested by the control computer. For these experiments, the GPS timing unit of the data acquisition system was used. The signal from the DAC then passes through an amplification stage to set the signal levels for application to the electrostatic actuators mounted behind the main test masses.

The differential arm-length change achieved by a particular injection depends on the calibration factor of the actuators used, in this case, the electrostatic drives. While this factor 
Table 1. Summary of injected signals for the three injection periods.

\begin{tabular}{lccc}
\hline & First period & Second period & Third period \\
\hline Time span (UTC) & $16 / 1 / 04,23: 22$ & $16 / 1 / 04,23: 52$ & $17 / 1 / 04,02: 56$ \\
& $-16 / 1 / 04,23: 51$ & $-17 / 1 / 04,02: 55$ & $-17 / 1 / 04,04: 35$ \\
No. of injected signals & 39 & 150 & 150 \\
Central frequency $f_{0}(\mathrm{~Hz})$ & 1080 & 1080 & 723 \\
Amplitude $A$ (arbitrary units) & 35 & 20 & 35 \\
\hline
\end{tabular}

is known, the injected signals were so far not calibrated to absolute units prior to the analysis and so only a relative comparison of the amplitudes of the detected to the injected signals is treated in this paper.

\subsection{Summary of injected events}

The injected events were grouped into three distinct populations. These are listed in table 1 . All injected events had a $Q$ of 9 .

\section{Search algorithms}

The search for burst-like gravitational wave signals is, in some sense, the most difficult of the gravitational wave searches currently underway. This is because the number of modelled burst sources is very small, and even then, these are not well understood. Therefore, the search needs to focus on unmodelled sources which in principle means that search algorithms need to be many, or general in their approach.

For this experiment, two search algorithms were chosen that search for arbitrary shortduration signals in the time/frequency plane. The two methods are called HACR and Excess Power.

Prior to the hardware injections, Excess Power had never been used to process GEO data. Therefore, using it to analyse the hardware injections provided, at a minimum, a demonstration that the algorithm could be applied to GEO data. On the other hand, HACR was used because it had previously been used to process GEO data and we had some experience in using the algorithm. HACR could therefore serve as a guide for the performance of the Excess Power algorithm. Moreover, its infrastructure was designed for quick analysis of GEO data.

Descriptions of the algorithms follow, together with details of the particular implementation and parameter tunings used to analyse the hardware injection data. The input parameters of both algorithms were tuned and fixed without prior knowledge of the injected events.

\subsection{HACR pipeline}

The HACR algorithm is an example of a class of time/frequency algorithms used to detect weak, short-period bursts in a noisy time series; it is an adaptation of the TFCluster algorithm described in [17]. An overview of the HACR pipeline is given in [18]; the mathematical details of the algorithm are described below. 
3.1.1. HACR algorithm. The time frequency representation that we use is called the spectrogram, $\rho(t, f)$, and is defined as

$$
\rho(t, f)=\left|\int_{-\infty}^{\infty} x\left(t^{\prime}\right) w\left(\left|t-t^{\prime}\right|\right) \mathrm{e}^{2 \pi \mathrm{i} f t^{\prime}} \mathrm{d} t^{\prime}\right|^{2},
$$

where $t$ is the time, $f$ is the frequency, $x(t)$ is the time series being analysed and $w(t)$ is a window function. A window function is typically non-zero only in a small interval $(\tau)$ around the origin. This implies that the contribution to the integral in the equation above comes only from a small interval around the time of interest. In physical terms, the spectrogram $\rho(t, f)$ represents a two-dimensional energy density function. Once the spectrogram is constructed, we identify regions in the spectrogram where the power is larger than some chosen threshold. Each such contiguous region is then labelled as an event.

For the purpose of analysis, the data are divided into segments of a convenient duration, $T$, of approximately a few seconds. Each data segment is then divided into windowed subsegments of duration $\tau$, and a discrete version of the spectrogram is constructed. The discrete spectrogram is represented by the two-dimensional set of numbers (referred to later as pixels) $\rho_{j k}$, where the indices $j$ and $k$ increase uniformly with time and frequency, respectively. Since each subsegment size is $\tau$, the frequency resolution, $\Delta f$, is therefore equal to $1 / \tau$. The resolution in time domain is $\tau-o$ where $o$ is the overlap between consecutive subsegments.

The next stage of the algorithm is to examine the discrete spectrogram and identify pixels with abnormally large power which one would not statistically associate with noise alone. The mean and variance of the spectrogram pixels is computed for each frequency bin and the pixels are normalized with respect to this mean and variance. There are two criteria applied while clustering pixels: first, each pixel in the cluster must have a normalized power higher than a lower threshold, $t_{1}$, and at least one pixel in the cluster must have a power larger than the upper threshold, $t_{\mathrm{u}}$.

Once the clusters have been identified the properties of the cluster are recorded. The duration and bandwidth of the cluster are simply defined by the extent of the cluster in the spectrogram in both time and frequency. The 'central' frequency and 'time of occurrence' of the event are determined by the weighted average of the pixel coordinates. The maximum pixel power, as well as the normalized power, is also recorded. In addition we also store the number of pixels in the cluster.

3.1.2. Choice of algorithm parameters. The HACR algorithm requires several parameters to be set, both for the construction of the spectrogram and for the clustering of pixels. The timescale, $T$, should be such that the noise is stationary over this time scale and it should be much larger than any burst timescale to allow us to get a good estimate of the average properties of the noise around a possible burst event. In this analysis, $T$ was chosen to be $16 \mathrm{~s}$ since on astrophysical grounds we would not expect gravitational wave burst events to last for more than a few milliseconds. The window width should be close to the burst time scale in order to maximize the ratio of signal power to noise power. On the other hand, we would like to retain as high a frequency resolution as possible. Considering both of these requirements, we chose a value of 512 samples (or 1/32 s) for $\tau$. The 'Hanning' window is used for the construction of the spectrogram. Consecutive subsegments are overlapped by as much as $80 \%$ to compensate for the attenuation by the window function. The upper and lower normalized power thresholds, $t_{\mathrm{u}}$ and $t_{1}$, were chosen to be 200 and 20 , respectively. Since the dynamic range of the data is quite large, the data were filtered through a time domain high-pass IIR filter with a lower cutoff frequency of $50 \mathrm{~Hz}$. This was necessary since we are computing 
the Fourier transform of very short stretches of data and the Hanning window does not have sufficiently good side-lobe suppression.

\subsection{Excess Power pipeline}

The Excess Power algorithm is described in [19, 20]. Like HACR, Excess Power is an algorithm that attempts to identify statistically significant bursts of excess power in the time/frequency domain. For completeness, a brief overview of this algorithm will be reviewed in this section.

The first step is to create a time/frequency map of the data. This is then tiled with sets of $N$ tiles, where $N$ is traditionally chosen to be a power of 2 in order to maximize computational efficiency. The time and frequency spans are split into $N_{t}=2^{i}$ and $N_{f}=2^{j}$ sections such that $2^{i+j}=N_{t} \times N_{f}=N$. For each time/frequency tile, the total power within the tile is computed by summing the power in each time/frequency bin within the boundaries of the tile. We define the total power in each tile as $P_{\mathcal{T}}$.

For stationary, Gaussian noise, the values of $P_{\mathcal{T}}$ follow a chi-squared distribution with $2 N_{t}$ degrees of freedom. Therefore, the excess power in each tile is calculated by

$$
\Delta P_{\mathcal{T}}=P_{\mathcal{T}}-\left\langle P_{\mathcal{T}}\right\rangle=P_{\mathcal{T}}-2 N_{\mathcal{T}} .
$$

From the distribution of $P_{\mathcal{T}}$, one can assign a confidence, $\alpha$, to the power observed in each tile. By then applying a threshold to $\alpha$, we can extract the tiles with statistically significant excess power in them as possible event candidates. Central frequencies are estimated by the frequency of the centre of the tile.

For the results presented in section 4.2, the Excess Power code processed the hardware injection data in the band between 500 and $1500 \mathrm{~Hz}$. This frequency range was chosen because this is the frequency region where GEO 600 was most sensitive during the S3 run. The data were initially split into sub-segments of 16384 samples (1 s) in length. A confidence threshold of $10^{-7}$ was used to select the triggers. Because GEO data are stored in double precision, it was necessary to cast it into single precision so that it could be processed by the Excess Power code (due to limitations of the implementation of the algorithm). Before casting the data to single precision, a high-pass filter with a corner frequency of $300 \mathrm{~Hz}$ was applied. For each trigger, the Excess Power algorithm gives an estimate of the relative excess power together with the frequency and time at which the signal power peaks.

\section{Results}

This section presents the results from running the HACR and Excess Power algorithms over the data containing the hardware injected signals. For both analyses, some discussion is given over to parameter estimation and algorithm performance. Both algorithms use a power signal-to-noise ratio to provide a measure of the strength of the detected signal: in the case of HACR, this is defined as the ratio of the peak power in a detected event to the average power at that frequency; for Excess Power, it is defined as the ratio of the detected excess power to the average power in the time-frequency tile of the given data segment.

\subsection{Results of the HACR pipeline}

We now describe the results obtained by analysing the data with the HACR algorithm. Two of the estimated parameters for the detected events as a function of time are presented in figure 1. The top panel on the graph is a scatter plot of the events on the time/frequency plane and the 

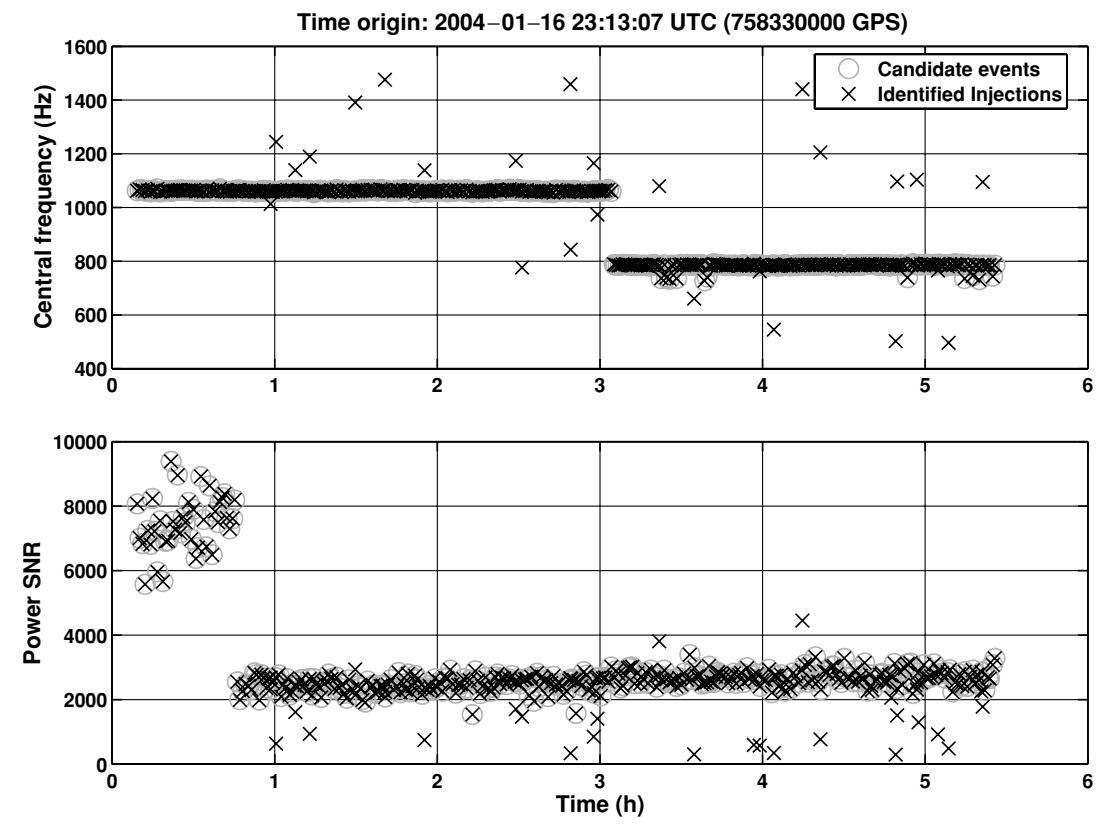

Figure 1. A plot showing two of the estimated output parameters of the HACR algorithm as a function of time. Also shown are those events that were subsequently identified with hardware injection events. The discrepancy of the population centred around $800 \mathrm{~Hz}$ from the injected events which had a central frequency of $723 \mathrm{~Hz}$ (see table 1), is discussed later in the text.

bottom panel is a scatter plot with the time of occurrence versus the relative power (to the upper threshold). The points marked as a cross indicate the events that were detected. Some of these events correspond to the hardware injected signals and the rest are presumed to be artefacts of the noise.

Since we have the list of times at which the signals were injected we can carry out a coincidence analysis to determine which of the detected events correspond to injected signals. In order to take into account possible errors in the detection times introduced by the algorithm, we allow for a time window of $100 \mathrm{~ms}$ around the injected events. If a detected event lies within this window it is associated with that particular injection. Such events are denoted with circles in figure 1. A total of 371 events were detected during the injection period. All 339 injected events are matched by a detected event close by. The remaining events which do not correspond with the injected signals are artefacts of noise. By looking at the two subplots together, three populations of injected events must be discussed.

4.1.1. Estimation of signal parameters. We now compare the parameters of the detected signals with those of the injected signals. The HACR algorithm does not assume any form for the signal and stores only generic properties of each event such as bandwidth, duration, central frequency. However, simple relationships between parameters of an assumed model or signal waveform and the properties of a HACR event can often be derived. In what follows we will illustrate this for each of the parameters of the injected sine-Gaussian signals.

The time of injection will be defined as the time when the sine-Gaussian reaches its peak. Figure 2 illustrates the timing offset of the detected events from the injected events. For the first two populations of injections at $1080 \mathrm{~Hz}$, the timing error is very small with a positive 


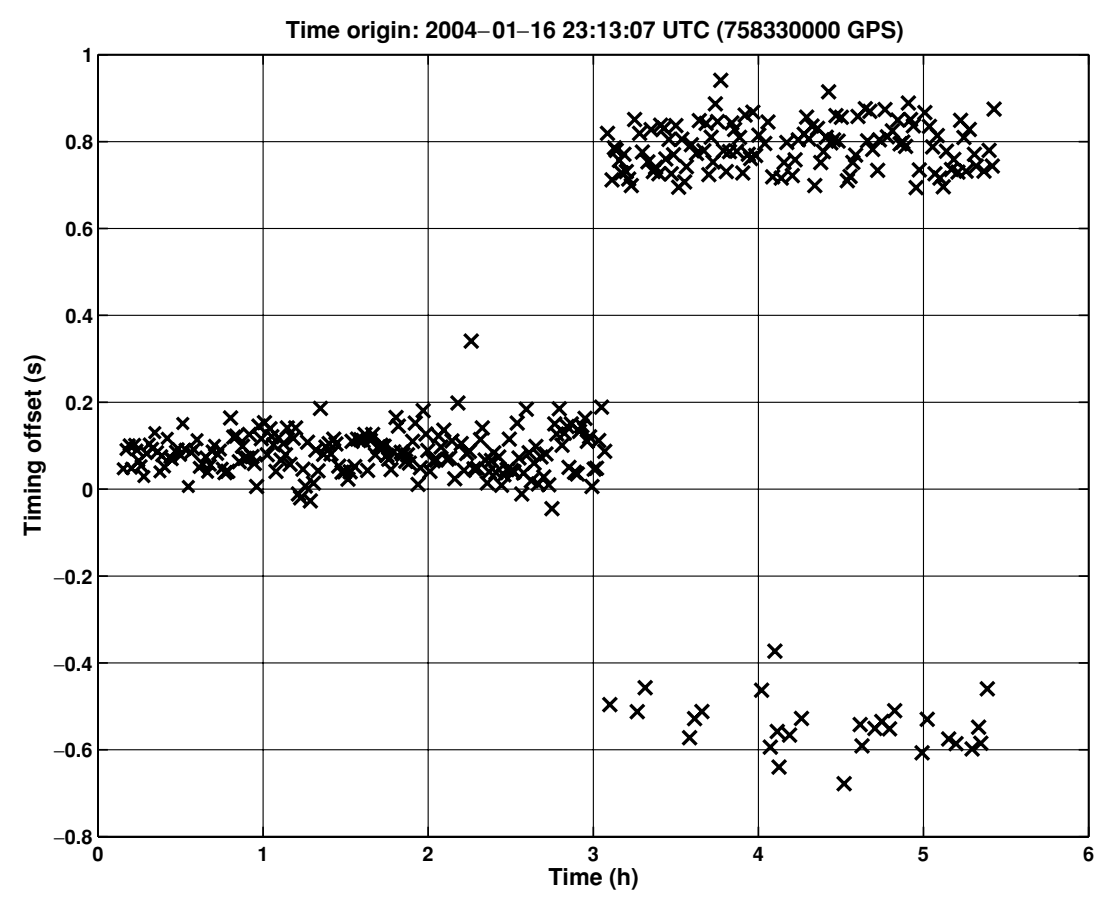

Figure 2. A figure showing the timing offset of the detected events from the injected events. In all cases, the offset is dominated by the errors introduced by the algorithm in determining the event times.

offset of a few samples $(0.1 \mathrm{~ms})$ which can be ascribed to the delay caused by the high-pass filter used to pre-process the data. The standard deviation of the error of the timing estimate is also very small, around $0.1 \mathrm{~ms}$.

For the third population of events there seems to be a large offset in the timing estimate. This offset can be ascribed to the way in which signal injection times were determined. The recorded injection signal was used to more accurately determine the injection time of each event. The HACR algorithm determines the time of occurrence of the event by computing a weighted sum of the pixels as described earlier. This method turns out to be quite accurate and HACR always picks the time corresponding to the central peak which corresponds to the time of occurrence.

The injection time, $t_{0}$, is defined as the time of the peak of the signal. Since the signal waveform is symmetric about $t_{0}$, taking the maximum of the absolute value of the signal should yield the correct time. However, if an offset is added to the signal (as is the case when recording signals in the DAQ system), this method can identify one of the two neighbouring peaks of the waveform, depending only on the noise in the signal. The offset was not accounted for in computing the injection times and as such, this effect shows up in the comparison of the injected to detected events. In particular, the effect is only present for an odd value of $f_{0}$ since the central peak in the waveform has negative amplitude in this case. This effect could be minimized in future by ensuring the recording of the injection signals uses more of the dynamic range of the input to the data acquisition system, thus reducing the size of any dc offset relative to the signal size.

It can be seen from figure 2 that the larger offsets correspond to half a cycle at the injection frequency $(723 \mathrm{~Hz}$, or $\pm 1.4 \mathrm{~ms})$. 


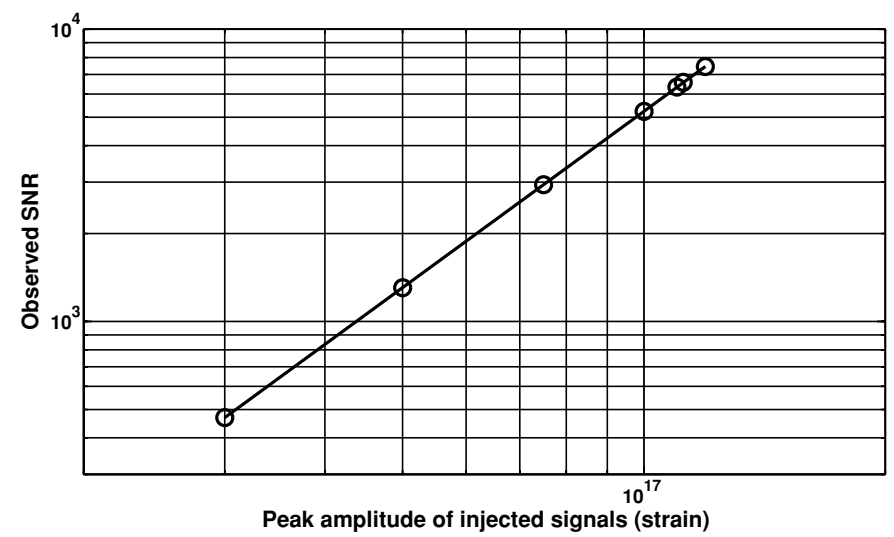

Figure 3. The relationship between the peak amplitude of a software-injected signal and the relative power estimated by HACR.

We next consider the estimation of the peak amplitude of the injected signals. The HACR algorithm works with the spectrogram whose pixels can be interpreted as the energy per unit time per unit frequency. It is difficult to find a direct quantitative relationship between the observed pixel amplitudes and the peak amplitude of the injected signals. In general we would expect the relative power computed by the HACR algorithm to be proportional to the square of the peak amplitude of the signal. This is borne out by simulations whose results are summarized in figure 3 . We carried out software injections using a subset of the data during the injection period. (We of course avoided the exact times where hardware injections took place.) The software injections were such that the observed relative power was in a range that included that observed while analysing the hardware injections. Using figure 3 it is simple to read off the peak amplitude corresponding to a specific observed relative power.

It can be seen from figure 1 and table 1 that the central frequency of the sine-Gaussians is estimated to within a few per cent. In other words, the central frequencies of the events recovered by the HACR algorithm agree with the central frequencies of the injected events to within $10 \%$. However, in all three populations there seems to be a bias in the observed frequency. In the first two populations the frequency is underestimated, the means of the observed frequencies being $1075 \mathrm{~Hz}$ and $1065 \mathrm{~Hz}$ respectively whereas the injected signals had a frequency of $1080 \mathrm{~Hz}$. In the third population of events, the frequency is overestimated and the bias is about $60 \mathrm{~Hz}$. The reason for this is not well understood. However, it must be recalled that the frequency resolution of the HACR algorithm is $32 \mathrm{~Hz}$.

\subsection{Results of the Excess Power pipeline}

For Excess Power, a low threshold was chosen and the injected signals were identified by taking the maximum trigger within a $\pm 1 \mathrm{~s}$ window of the injection time and a $\pm 50 \mathrm{~Hz}$ frequency window around the injection frequency; the idea of using a low threshold here was to focus on testing the parameter estimation of the Excess Power algorithm, not so much on detection efficiency. Figure 4 plots the normalized excess power and central frequencies of the triggers found above a confidence threshold of $10^{-7}$. Marked in dark circles are the triggers that are identified as injections using the conditions mentioned above. The large normalized excess powers of the injected signals make them clearly visible above the background events. While 

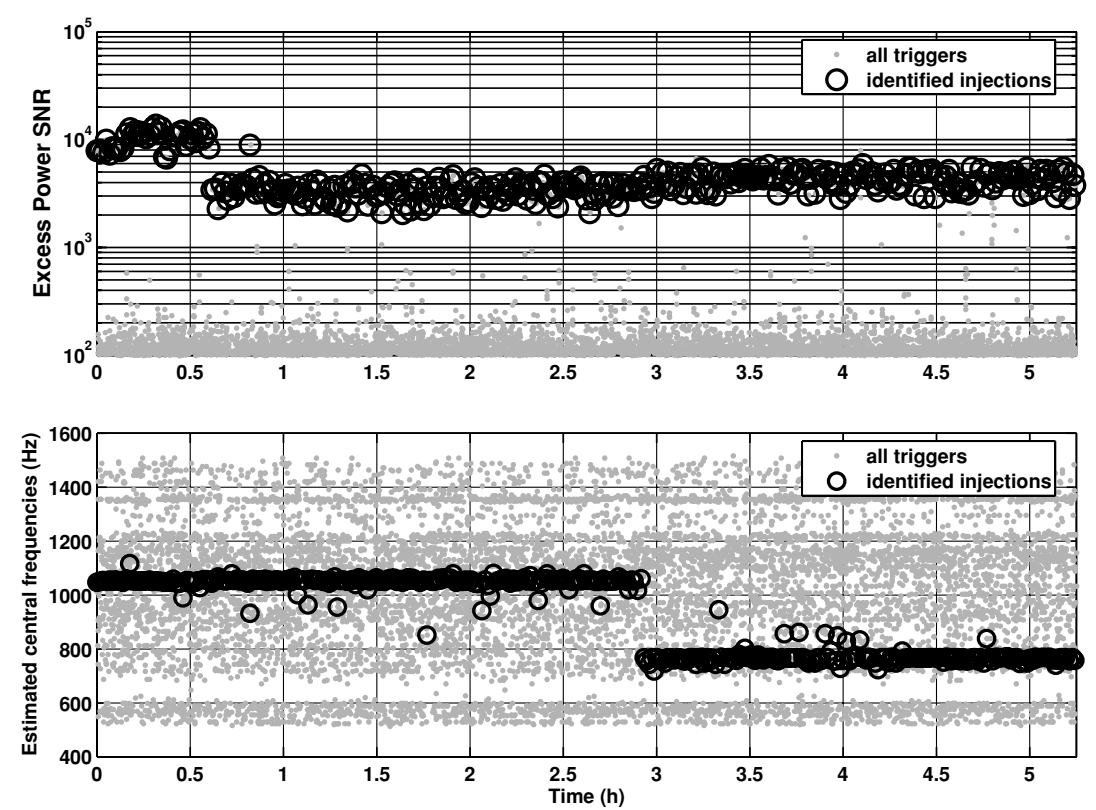

Figure 4. A scatter plot of the SNR and central frequencies of triggers observed over the injection period.

the injected signals are less visible against the large background in the central frequency plot, the identified triggers show a rather clear trend.

4.2.1. Estimation of signal parameters. A study was made of how well the Excess Power algorithm can estimate the time at which the signal peaks (which will be referred to as peak time from here on). Given the injection times, we calculate the standard deviation of the difference between the injection times and the peak times. This measure (which will be referred to as timing uncertainty from here on) gives us an idea of how much scatter there is in the peak time estimate as performed by Excess Power, as well as highlighting any systematic offsets in the peak time estimates.

The timing uncertainty has direct consequences on how wide a time window should be set in a search for coincidence triggers between multiple detectors - a technique used to corroborate the detection of burst gravitational wave signals. The larger the timing uncertainty, the wider the required coincidence window which leads to a larger number of false alarms. We obtain a value of $0.13 \mathrm{~s}$ which is extremely large, given the huge amplitudes of the injected signals. Moreover, upon further investigation, we observed that the timing uncertainty is linked to the length of the data subsegments.

Figure 5 plots the time difference between the injection times and the peak times for different subsegment lengths. One can see that the timing uncertainty is directly proportional to the subsegment length. This was because the minimum time width of the Excess Power tiles is proportional to the subsegment length. The smaller subsegment lengths mean we have a better time resolution and thus have smaller tiles. In figure 5, we can see that for a subsegment length of 4096 samples, the timing uncertainty for the hardware injected signals was about $40 \mathrm{~ms}$. This value is still about 40 times larger than that obtained using HACR. We were not able to reduce the subsegment lengths any further because the smaller tile sizes were too 


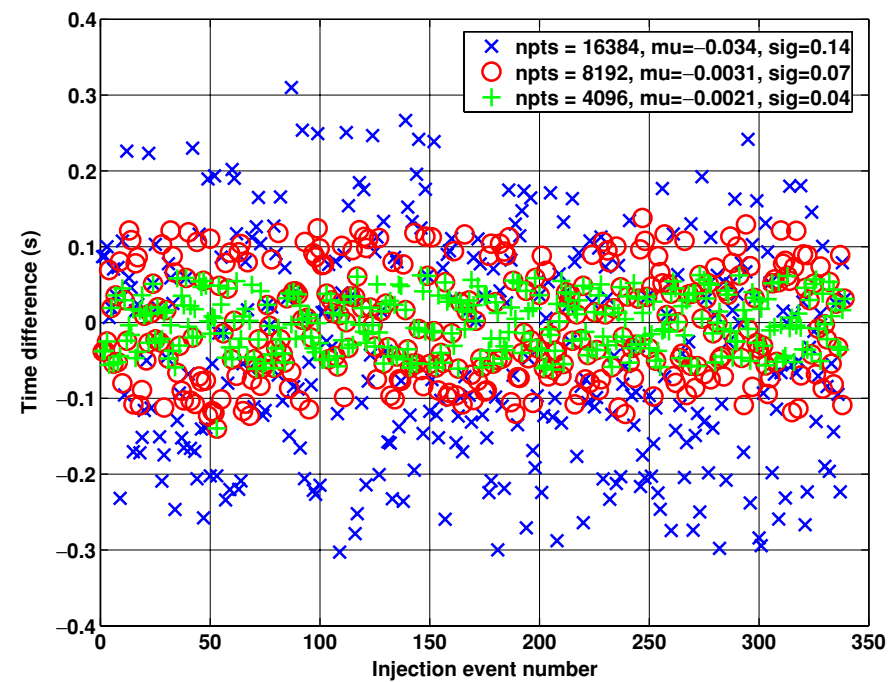

Figure 5. Scatter plot of the time difference between the injected events and the peak times of the observed events for different subsegment lengths. The mean of each population is labelled 'mu' in the caption, and the standard deviation, 'sig'.

(This figure is in colour only in the electronic version)

small for any one tile to encompass the injected signals, causing the normalized excess power estimates to be reduced.

Another reason for the large timing uncertainties observed for Excess Power was the choice to define the centre of the tile as the peak time estimate. Figure 6 gives an example of how this definition of peak time leads to a large timing uncertainty. Because the duration of the tile is larger than the injected signal, the peak time defined at a large offset from the time the injected signal occurred.

As with the HACR analysis, sine-Gaussians of different amplitudes were injected directly into the data in the software to quantify the normalized excess power units in terms of strain. Figure 7 plots the normalized excess power for the different software-injected signal amplitudes.

Finally, the central frequency estimated by Excess Power was compared to the injected values. In the first two periods, the mean observed central frequencies were $1051 \mathrm{~Hz}$ and $1049 \mathrm{~Hz}$ while the central frequency of the injected signals was $1080 \mathrm{~Hz}$. In the third period, the mean observed central frequencies were $768 \mathrm{~Hz}$ as compared to $723 \mathrm{~Hz}$ for the injected signals. Again we see an over-estimate of the central frequency of the third population of events due to the bias in the selection of the injected time (the later peak is always picked) caused by a dc offset in the data acquisition system.

\subsection{Pipeline comparison}

Table 2 shows a comparison of the parameter estimations from the two pipelines. The mean injected and detected powers for the three injection periods have been normalized to the first period. It should be noted that, although the injected amplitude of the first and the third injection periods was the same, the central frequency was reduced resulting in a lower signalto-noise ratio for the injected events. Since the two search algorithms do not estimate absolute 


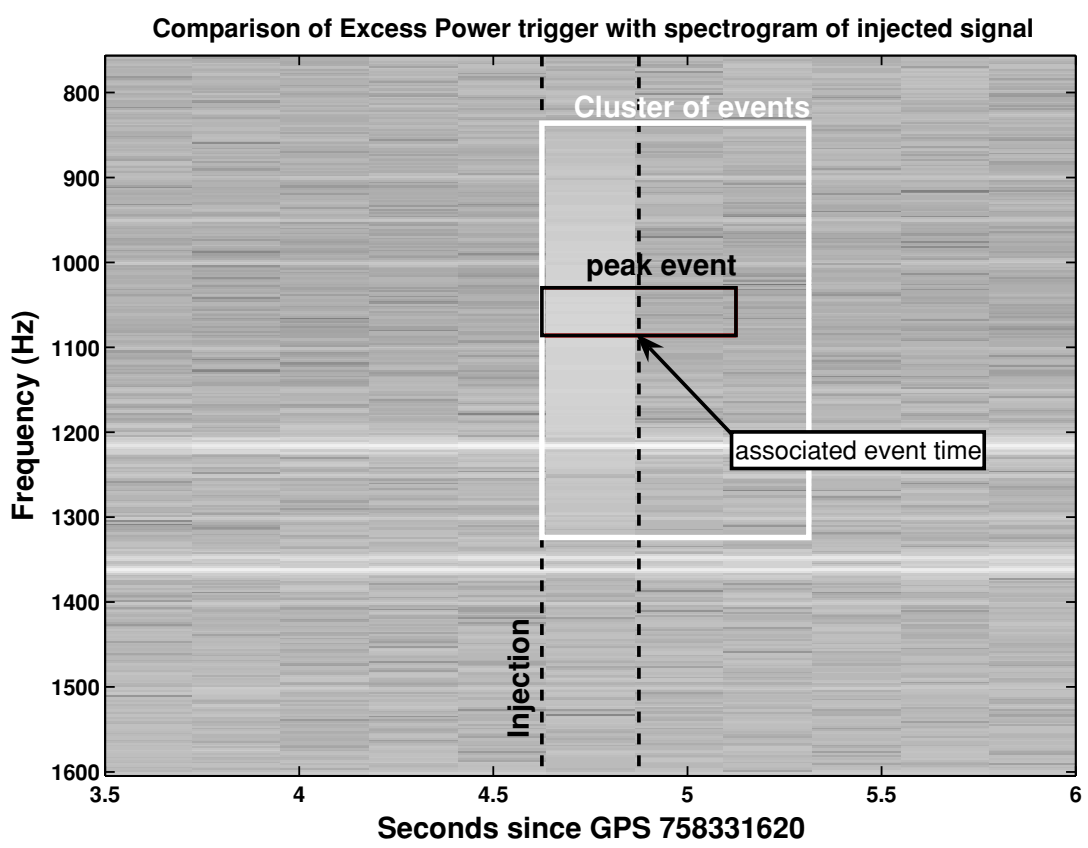

Figure 6. Comparison of the peak time as estimated by Excess Power with a spectrogram of the actual signal. The injected signal is the light stripe that spans a few hundred $\mathrm{Hz}$; it is shown bounded by the two vertical dashed lines. For such large injected signals, many events are generated. These are clustered and the peak event (largest SNR) is selected from the cluster.

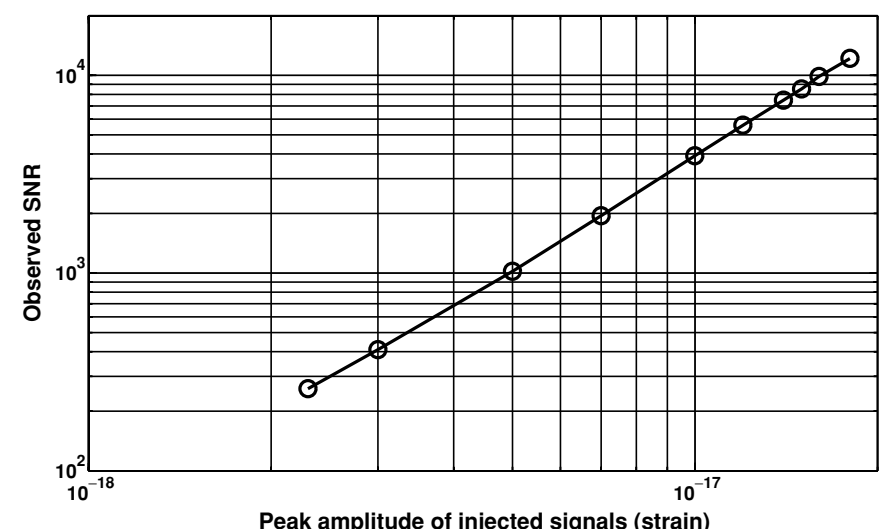

Figure 7. The observed SNR for different sine-Gaussian amplitudes injected via software.

power but instead some measure of signal-to-noise ratio, we see values far from 1.0 for the mean detected amplitudes of the third period injections.

\section{Summary}

Hardware injections of short-duration sine-Gaussian waveforms were performed at GEO 600 for the first time. Two different burst-search algorithms were used to analyse 
Table 2. Comparison of parameter estimations of detected events. The mean power estimations are normalized to the mean power determined for the first series of injections; this allows the injected signal power to be compared relatively to the detected signal powers for the two algorithms.

\begin{tabular}{lccc}
\hline & First period & Second period & Third period \\
\hline Time span (UTC) & $16 / 1 / 04,23: 22$ & $16 / 1 / 04,23: 52$ & $17 / 1 / 04,02: 56$ \\
& $-16 / 1 / 04,23: 51$ & $-17 / 1 / 04,02: 55$ & $-17 / 1 / 04,04: 35$ \\
Injections & & & \\
$f_{0}(\mathrm{~Hz})$ & 1080 & 1080 & 723 \\
$N$ events & 39 & 150 & 150 \\
$A^{2}$ (normalized) & 1.0 & 0.33 & 1.0 \\
& & & 768 \\
Excess Power & 1051 & 1049 & 150 \\
Mean $f_{0}(\mathrm{~Hz})$ & 39 & 150 & 0.43 \\
$N$ detections & 1.0 & 0.34 & 789 \\
Mean normalized power & & & 150 \\
& 1075 & 1065 & 1.3 \\
HACR & 39 & 150 & \\
Mean $f_{0}(\mathrm{~Hz})$ & 1.0 & 0.29 & \\
$N$ detections & & & \\
Mean normalized power & & & \\
\hline
\end{tabular}

the data containing the injected events. All injected events were identified by both algorithms. However, the ability of the algorithms to properly estimate the underlying waveform parameters varied for the different parameters.

The event times were best estimated by the HACR algorithm which achieved an accuracy of around $1 \mathrm{~ms}$. The Excess Power algorithm uses the central time of the (relatively large) tile in which the event is located. This leads to larger possible errors. Efforts are being made to improve the way the algorithm estimates the time of an event. The central frequency was estimated equally well by both algorithms, with Excess Power having a slightly larger bias than HACR. The accuracy of the central frequency estimates was around $30 \mathrm{~Hz}$ or more for both.

The estimation of signal power or amplitude is difficult due to the way that the individual algorithms define the power in the signal. Nevertheless, some comparisons were performed but this needs further exploration to be useful. For example, the induced strain of the injected signals could be determined and used to compare with the detected events.

This set of experiments has proven useful in identifying possible sources of systematic and random errors inherent to the algorithms tested. Further hardware and software injections should be performed in the future to confirm and attempt to rectify these sources of error.

\section{Acknowledgments}

We would like to thank Patrick Brady and Saikat Majumder from the University of Milwaukee for the help and support provided to get the Excess Power code to work with GEO data.

\section{References}

[1] Heng I S, Daw E, Giaime J, Hamilton W O, McHugh M P and Johnson W W 2002 Allegro: noise performance and the ongoing search for gravitational waves Class. Quantum Grav. 19 1889-95

[2] Zendri J-P et al 2002 Status report and near future prospects for the gravitational wave detector AURIGA Class. Quantum Grav. 19 1925-33 
[3] Astone P (ROG Collaboration) 2004 Seven years of data taking and analysis of data from the Explorer and Nautilus gravitational wave detectors Class. Quantum Grav. 21 S1585-94

[4] Willke B et al 2004 Status of GEO 600 Class. Quantum Grav. 21 S417-23

[5] Sigg D 2004 Commissioning of LIGO detectors Class. Quantum Grav. 21 S409-15

[6] Takahashi R and the TAMA Collaboration 2004 Status of TAMA300 Class. Quantum Grav. 21 S403-8

[7] Acernese F et al 2004 Status of VIRGO Class. Quantum Grav. 21 S385-94

[8] Arnaud N, Barsuglia M, Bizouard M-A, Brisson V, Cavalier F, Davier M, Hello P, Kreckelbergh S, Porter E K and Pradier T 2003 Comparison of filters for detecting gravitational wave bursts in interferometric detectors Phys. Rev. D 67062004

[9] Klimenko S, Yakushin I, Rakhmanov M and Mitselmakher G 2004 Performance of the WaveBurst algorithm on LIGO data Class. Quantum Grav. 21 S1685-94

[10] McNabb J W C, Ashley M, Finn L S, Rotthoff E, Stuver A, Summerscales T, Sutton P, Tibbits M, Thorne K and Zaleski K 2004 Overview of the BlockNormal event trigger generator Class. Quantum Grav. 21 S1705-10

[11] Hewitson M et al 2003 Calibration of the power-recycled gravitational wave detector, GEO 600 Rev. Sci. Instrum. 744184

[12] Gossler S et al 2002 The modecleaner system and suspension aspects of GEO 600 Class. Quantum Grav. 19 1835-42

[13] Plissi M V, Torrie C I, Husman M E, Robertson N A, Strain K A, Ward H, Lück H and Hough J 2000 GEO 600 triple pendulum suspension system: seismic isolation and control Rev. Sci. Instrum. 71 2539-45

[14] Kötter K et al 2002 Data acquisition and detector characterisation of GEO 600 Class. Quantum Grav. 20 1399-407

[15] Kötter K et al 2004 Timing accuracy of the GEO 600 data acquisition system Class. Quantum Grav. 21 $493-500$

[16] Hewitson M et al 2004 Calibration of the dual-recycled GEO 600 detector for the S3 science run Class. Quantum Grav. 21 S1711-22

[17] Sylvestre J 2002 Time-frequency detection algorithm for gravitational wave bursts Phys. Rev. D 66102004

[18] Heng I S, Balasubramanian R, Sathyaprakash B S and Schutz B F 2004 First steps towards characterizing the hierarchical algorithm for curves and ridges pipeline Class. Quantum Grav. 21 S821

[19] Anderson W G et al 2001 An excess power statistic for detection of burst sources of gravitational radiation Phys. Rev. D 63042003

[20] http://www.lsc-group.phys.uwm.edu/lal/lsd.pdf 